\title{
Cardiac troponin testing for diagnosis of acute coronary syndromes in primary care
}

\begin{abstract}
1 cute coronary syndromes $\triangle$ (ACS) are a leading cause of illness and death in Australia. Around 75000 Australians are hospitalised for ACS each year, with $\$ 8$ billion spent annually on related inpatient care. ${ }^{1}$ While mortality caused by ACS is declining because of better control of coronary risk factors and the introduction of new treatments, ${ }^{2}$ at least 10000 Australians still die each year as the result of ACS. ${ }^{1}$
\end{abstract}

The spectrum of ACS includes unstable angina, where atherosclerotic plaque rupture leads to arterial occlusion and myocardial ischaemia, and myocardial infarction, where ischaemia progresses to myocardial cell necrosis. Further classification into ST elevation myocardial infarction (STEMI) and non-ST elevation myocardial infarction (NSTEMI) is based on electrocardiographic (ECG) findings. Overall, the rate of inhospital major adverse cardiac events caused by ACS (death, cardiac arrest, recurrent myocardial infarction, worsening heart failure, major bleeding or stroke) approaches $30 \%$ for STEMI and 20\% for NSTEMI. ${ }^{3}$ Patients with unstable angina are also at increased risk of death and subsequent myocardial infarction, even in the absence of myonecrosis. ${ }^{4}$

Diagnosing ACS is challenging in primary care as well as in the tertiary setting; $15 \%$ of patients who experience an ACS initially contact their general practitioner. ${ }^{5}$ The diagnosis of ACS in primary care is not always straightforward; signs and symptoms alone are neither sensitive nor specific in the prehospital population, ${ }^{6}$ and the validity of clinical prediction rules for ACS in primary care populations is limited.

Given these limitations, there are potential benefits to using cardiac biomarkers in primary care. Cardiac troponin (cTn) is the main biomarker in patients who present with possible doi: 10.5694/mjal4.01154

\section{Abstract}

Objective: To examine the use of cardiac troponin (cTn) testing for acute coronary syndrome (ACS) diagnosis in primary care.

Design and setting: Prospective cohort study; general practitioner-initiated cTn tests conducted from 24 September 2009 to 3 September 2010 in Perth, Western Australia. Patient outcomes were obtained from linked data sources for up to 12 months after the final test. Clinical information and outcomes were compared with data from emergency department patients with ACS symptoms.

Participants: 369 patients with samples collected at community laboratories. Requesting GPs provided the clinical context for testing.

Main outcome measures: Cardiovascular risk status, symptoms prompting cTn testing; estimated ACS likelihood and referral decision before and after testing; result turnaround time; hospital presentations, procedures and mortality.

Results: Of the 328 GPs who received a survey request, 124 (37.8\%) responded. 122 of 124 test results (98.4\%) were negative. Based on clinical risk factors, 71 of 104 patients $(68.2 \%)$ were at high or intermediate risk of ACS. 69 of 124 patients (55.6\%) had typical ischaemic pain and 62 of 124 patients $(50.0 \%)$ were tested within 48 hours of symptom onset (23.4\% within 12 hours, with no serial testing). Test results affected GPs' estimation of ACS likelihood $(P<0.01)$ but not their referral decisions $(P=$ 0.23). 94 of 355 patients (26.5\%) presented to hospital with cardiovascular symptoms or diagnoses during follow-up; 27 of 355 patients (7.6\%) had at least one ACS, 13 of 255 (3.7\%) within 30 days of testing.

Conclusions: GP-initiated cTn testing involves patients at high risk of ACS. ACS and associated adverse outcomes can occur in patients undergoing testing, even when the cTn test result is negative. Potential gaps exist in physicians' understanding of the limitations of cTn testing, and cTn test results have minimal influence on their management of patients. GPs may benefit from guidance about ordering cTn testing.

myocardial necrosis with high sensitivity and specificity, and allows differentiation of myocardial infarction from unstable angina. ${ }^{8}$ Examples of the benefits of cTn as a biomarker in primary care include the diagnosis of myocardial infarction where it was not suspected initially because of atypical presenting features; the exclusion of myocardial infarction in low-risk patients; and the conservation of resources by avoiding hospital referral. ${ }^{9}$

However, there are pitfalls and practical considerations associated with cTn as a biomarker in primary care. Compared with those presenting directly to hospital, patients with ACS who first consult a community physician have longer prehospital delay $^{10}$ and decreased survival. ${ }^{11}$ Several authors have expressed concern that GP cTn requests contributes to these outcomes, ${ }^{12,13}$ and there is also evidence of overinterpretation of positive results ${ }^{12}$ and over-reliance on negative results. ${ }^{14}$ There can also be problems with follow-up if the test results are notified after normal practice hours.

In this study, we examined a population of patients with possible symptoms of ACS who underwent GP-initiated cTn testing. We compared the incidence of ACS and associated adverse outcomes with those in patients who had presented to hospital for cTn testing. We also explored GPs' knowledge of the limitations of the usefulness of cTn testing, and the influence of cTn test results on their diagnosis and hospital referral practices. 


\section{Methods}

\section{Study design, setting and participants}

This study employed a prospective cohort design. We recruited patients who had cTn blood tests ordered by a GP in a non-hospital setting, and who had their sample collected at the community collection centres of two of five pathology laboratories in urban Perth, Western Australia. The period of recruitment was 24 September 2009 - 3 September 2010. Patients with samples collected at rural and regional centres were excluded because it was considered likely that GPs in those areas would employ cTn testing differently to urban GPs. Additional exclusion criteria were: patients less than 18 years of age, cTn tests ordered by non-GP doctors, tests ordered for emergency department (ED) patients, and tests ordered by GPs who declined to participate in the study (Box 1).

Data sources and measurement GP cohort: laboratory data. A research assistant at each laboratory obtained consecutive cTn test results requested by GPs, using the practice address to establish GP status.

GP cohort: survey data. Laboratory research assistants approached requesting GPs for de-identified details about the clinical scenario leading to the cTn test request and the clinical course after notification of the result. GPs were contacted within 1 week of testing, with telephone follow-up to non-responders 1 week after the initial contact. Information was collected on a one-page survey sent and returned by fax, with an information sheet and consent form concurrently sent to the doctor. Risk stratification was undertaken using elements of the National Heart Foundation/Cardiac Society of Australia and New Zealand (NHF/ CSANZ) criteria that could be readily assessed during a general practice consultation. ${ }^{15}$

GP cohort: linked data. Linked data were obtained from the Department of Health Western Australia Data Linkage System (WADLS) for all patients for a minimum 12-month period after the date of their test, irrespective of whether their GP had responded to the survey. The final cTn test included in our study was performed in September 2010, and follow-up continued until October 2011. Outcomes were defined according to standardised definitions recommended for Australasian ACS research. ${ }^{16}$ Specific diagnosis and procedure codes were selected from the International Classification of Diseases, 10th revision,
Australian modification (ICD-10 $\mathrm{AM})^{17}$ and the Australian Classification of Health Interventions. ${ }^{18}$ Linkage and extraction were performed in November 2013 to compensate for delay in updating of Department of Health records. Records were excluded from analysis if no principal diagnosis was stated, or if the presenting symptom or principal diagnosis was insufficiently specific to allow classification. Duplicate records with more than one hospital admission for the same patient on the same day were treated as one admission for the purposes of statistical analysis.

ED cohort. Clinical presentations and outcomes in the GP survey cohort were compared with an ED cohort using the Multiple Infarct Markers in Chest Pain (MIMIC) study dataset. ${ }^{19}$ This prospective cohort study was conducted between September 2008 and June 2009 in two tertiary and three general hospitals in urban Perth. The urban catchment areas of the hospitals were similar to those of the collection centres in the GP survey cohort. Participants were a representative sample of patients undergoing evaluation for possible ACS with serial cTn testing. Patients were excluded if they were less than 18 years of age or pregnant, and where ECG criteria had indicated urgent reperfusion therapy.

1 Flow chart for inclusion of participants in the study (the GP cohort)

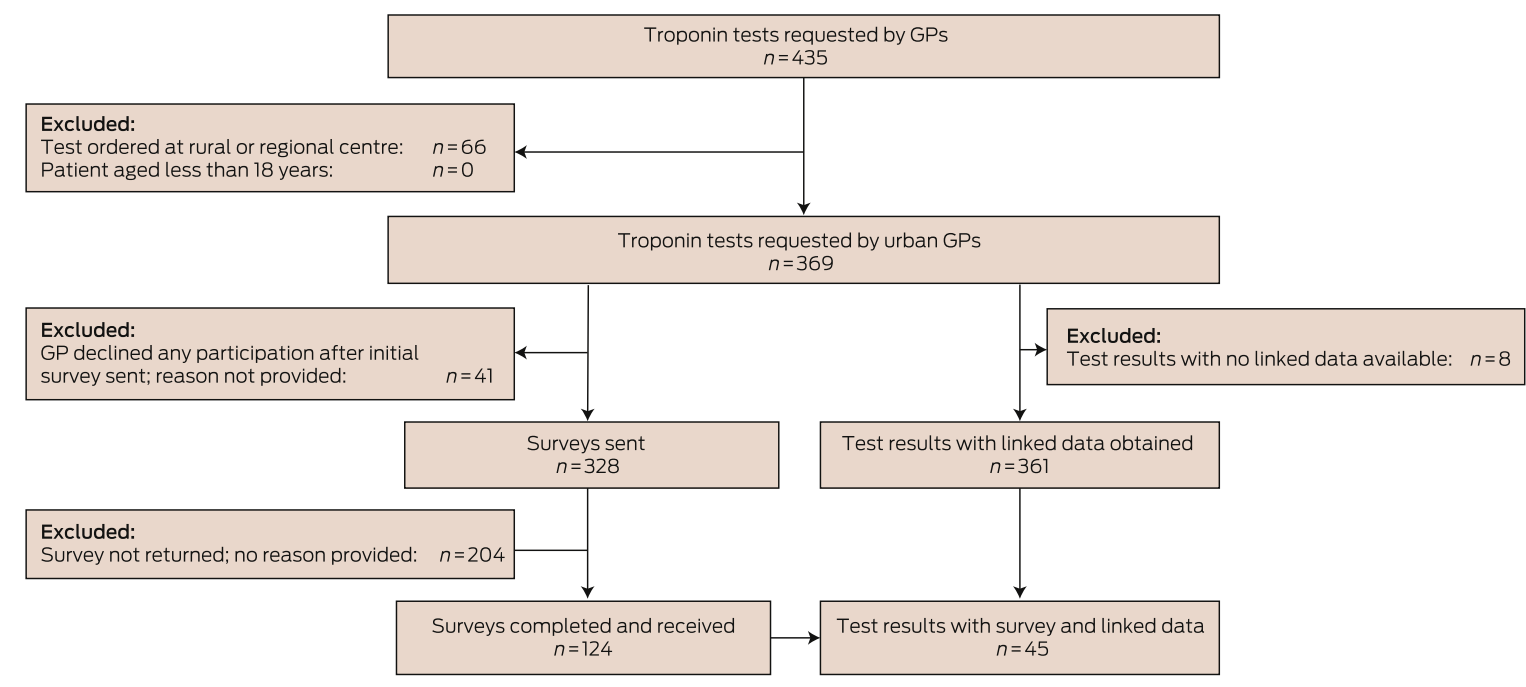




\section{Statistical methods}

All statistical analyses were performed with SAS version 9.4. Differences between group characteristics were assessed by two-sample $t$ test for continuous variables and by $\chi^{2}$ and Fisher exact tests where appropriate, based on expected frequencies for dichotomous variables. Statistical significance was defined as $P<0.05$.

\section{Ethics approval}

Ethics approval to conduct the survey was obtained from Human Research Ethics Committees of the University of Western Australia (RA/4/1/2275; 13 July 2009), St John of God Hospital (370; 7 May 2009), the Department of Health Western Australia (2013.04.02; 9 April 2013) and the South Metropolitan Health Services Board (08.136; 28 August 2014). The medical directors of the participating laboratories gave consent for the provision of laboratory data, and ethics approval was obtained from their institutional ethics committees (details available on request).

\section{Results}

\section{Participants}

Box 1 depicts participant flow through the study. There were no significant differences between included and excluded patients with respect to age or sex (each $P>0.10)$.

\section{Descriptive data}

Box 2 presents the characteristics of the 124 patients in the GP cohort for whom survey data were available. The most common presentation was pain typical of cardiac ischaemia $(55.6 \%)$.

Data on coronary risk factors were available for 104 GP cohort patients. Six patients $(5.7 \%)$ were at high risk of ACS according to the NHF/ CSANZ risk stratification framework, ${ }^{15}$ with a combination of typical symptoms and diabetes. A further 65 patients $(62.5 \%)$ were at intermediate risk of ACS, including 40 (38.5\%) over 65 years of age, 16 (15.4\%) with various combinations of hyperlipidaemia, a family history of

\begin{tabular}{|c|c|c|c|}
\hline Characteristic & $\begin{array}{l}\text { GP cohort } \\
(n=124)\end{array}$ & $\begin{array}{l}\text { ED cohort } \\
(n=1758)\end{array}$ & $P$ \\
\hline Median age, years (interquartile range) & $61(45-73)$ & $62(50-74)$ & 0.38 \\
\hline Sex (male) & 55 (44.4\%) & $984(56.0 \%)$ & $<0.01$ \\
\hline \multicolumn{4}{|l|}{ Cardiac troponin (cTn) test result* } \\
\hline Positive & $2(1.6 \%)$ & $168(10.7 \%)$ & $<0.01$ \\
\hline Negative & $122(98.4 \%)$ & $1408(89.4 \%)$ & $<0.01$ \\
\hline \multicolumn{4}{|l|}{ Risk factors $^{\dagger}$} \\
\hline Smoker ${ }^{\ddagger}$ & $15(12.1 \%)$ & $425(24.2 \%)$ & 0.01 \\
\hline Hypertension & $51(41.1 \%)$ & $923(52.5 \%)$ & 0.02 \\
\hline Dyslipidaemia & $47(37.9 \%)$ & $842(47.9 \%)$ & 0.03 \\
\hline Diabetes & $15(12.1 \%)$ & $327(18.6 \%)$ & 0.07 \\
\hline Past history of $\mathrm{CHD}$ or equivalent & $8(6.5 \%)$ & $621(35.3 \%)$ & $<0.01$ \\
\hline Family history of $\mathrm{CHD}^{\S}$ & $24(19.4 \%)$ & 879 (50.0\%) & $<0.01$ \\
\hline \multicolumn{4}{|l|}{ Presenting symptoms } \\
\hline Typical pain & $69(55.6 \%)$ & & \\
\hline Atypical pain & $27(21.8 \%)$ & & \\
\hline Non-pain symptoms & $24(19.4 \%)$ & & \\
\hline No symptoms" & $3(2.4 \%)$ & & \\
\hline Not recorded & $1(0.8 \%)$ & & \\
\hline \multicolumn{4}{|l|}{$\begin{array}{l}\text { Symptom duration at time of } \\
\text { presentation to GP }\end{array}$} \\
\hline Less than $12 \mathrm{~h}$ & $29(23.4 \%)$ & & \\
\hline $12 \mathrm{~h}-48 \mathrm{~h}$ & 33 (26.6\%) & & \\
\hline More than $48 \mathrm{~h}$ & 57 (46.0\%) & & \\
\hline Not recorded & $5(4.0 \%)$ & & \\
\hline \multicolumn{4}{|l|}{$\begin{array}{l}\text { Estimated glomerular filtration } \\
\text { rate }\left(\mathrm{mL} / \mathrm{min} / 1.73 \mathrm{~m}^{2}\right)\end{array}$} \\
\hline Less than 30 & $3(2.4 \%)$ & & \\
\hline $30-60$ & $19(15.3 \%)$ & & \\
\hline More than 60 & $63(50.8 \%)$ & & \\
\hline Not recorded & 39 (31.5\%) & & \\
\hline
\end{tabular}

ED = emergency department; CHD = coronary heart disease. *ED cohort: 1576 patients with 8 h-12 h cTn level data. † GP cohort: 104 patients with complete risk factor data. $\ddagger$ Current smoker or previous smoker of $>10$ pack-years. $\S$ First or second degree relative $<60$ years of age. $\uparrow$ Reasons for cTn: to investigate elevated creatine kinase levels; for monitoring of cardiac status while on statin; psychiatry patient at risk of cardiac complications of treatment.

coronary heart disease (CHD), smoking history and hypertension, and nine patients $(8.7 \%)$ with diabetes and atypical symptoms of ACS.

The median time from specimen collection to sample registration at the processing laboratory was 31 minutes (range, $0 \mathrm{~min}-1465 \mathrm{~min}$ ). This interval depended on the location of the collection centre; centres co-located with laboratories had the shortest intervals. Overall, the median time between specimen collection and availability of the test result was 128 minutes (range, 23min$1466 \mathrm{~min})$.

Before receiving the test results, most GPs $(80 / 124,64.5 \%)$ rated the likelihood of ACS in their patient as low (less than 5\%). This proportion increased after the results were received (to $110 / 124,88.7 \%$ ). A 
3 Flow chart of the outcomes for the GP cohort

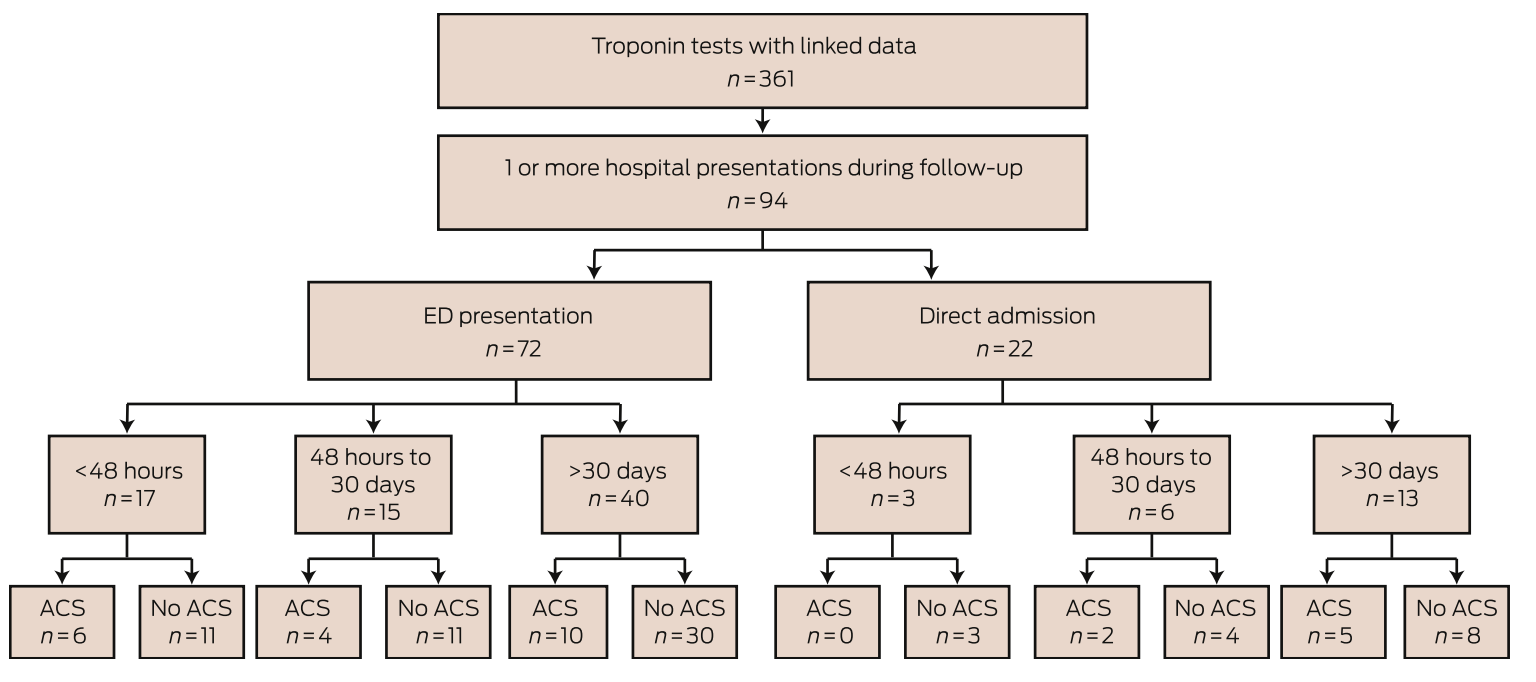

significant proportion of GPs (34/124, $27.5 \%$ ) changed their assessment of the likelihood of ACS after a negative test result $\left(\chi^{2}\right.$ test, $\left.P<0.001\right)$.

Most GPs (85/124, 68.5\%) intended to manage the patient themselves before receiving the test result, rather than referring them to a hospital or cardiology. This increased to 95/124 GPs $(76.6 \%)$ after the results were known. Despite the test result having a significant effect on the estimated likelihood of ACS, it did not significantly influence the intended management of the patient $\left(\chi^{2}\right.$ test, $P=0.23)$.

The prevalence of smoking $(P=0.01)$, hypertension $(P=0.02)$, dyslipidaemia $(P=0.03)$ and a personal or family history of CHD $(P<0.001)$ were all significantly greater in the GP survey group than in the MIMIC dataset cohort (Box 2).

\section{Outcome data}

GP cohort: linked data. Linked data were available for 361 tests performed for 355 patients; data for eight patients could not be linked because of insufficient identifying information.

There were 176 presentations to hospital with a cardiovascular symptom or diagnosis during follow-up, whether by presentation to an ED (112 presentations) or by direct admission (64 presentations). Of the
112 presentations to an ED in the GP cohort, 87 were assigned a triage category of 1 or 2 , indicating that they required medical review immediately or within 10 minutes.

In total, 94 of 355 of the GP cohort (26.5\%) presented at least once to a hospital during follow-up with cardiovascular diagnoses (Box 3 and Box 4). Twenty-one of these 94 patients (22.3\%) presented to a hospital within 48 hours of testing. The median time from testing to first presentation was 33 days (range, 0 days -551 days).
Within 48 hours of testing, six of the GP cohort $(1.7 \%)$ had been diagnosed with an ACS; the median time from specimen collection to hospital presentation for these patients was 382 minutes (range, $80 \mathrm{~min}-1312 \mathrm{~min}$ ). Box 5 lists the components of delay for this group.

Within 30 days of cTn testing, 13 of 355 patients in the GP cohort (3.7\%) had at least one ACS. Complications included one death from a cardiovascular cause (occurring outside of hospital within 1 week of the test, in a

4 Details of 94 hospital presentations by members of the GP cohort during follow-up

\section{Acute coronary syndrome}

Death outside hospital due to cardiovascular cause (1); cardiac arrest (1); cardiogenic shock (1); ST elevation myocardial infarction (1); non-ST elevation myocardial infarction (5); acute myocardial infarction (9); unstable angina (9)*

Coronary heart disease, not otherwise specified

Cardiomyopathy

Heart failure

Arrhythmia

Supraventricular tachycardia (1); ventricular tachycardia (1); atrial fibrillation (1); atrioventricular block, 2nd degree (1); bradycardia (1); cardiac arrhythmia, other (1)

Other cardiovascular diagnosis

Aortic valve stenosis (1); hypertensive (6); chest pain, anterior chest wall (3); chest pain on breathing (26); chest pain unspecified (4); syncope (1); dizziness (3); palpitations (2); dyspnoea (1)

* No hospital admission data were collected for four patients with unstable angina. 
5 Delay components in six patients presenting with acute coronary syndrome within 48 hours of a cardiac troponin (cTn) test

\begin{tabular}{|c|c|c|c|c|c|c|c|}
\hline $\begin{array}{l}\text { Age } \\
\text { (years) }\end{array}$ & $\begin{array}{l}\text { Collection to } \\
\text { registration (min) }\end{array}$ & $\begin{array}{l}\text { Registration to } \\
\text { result (min) }\end{array}$ & $\begin{array}{l}\text { Collection to } \\
\text { result (min) }\end{array}$ & Result & eGFR & $\begin{array}{l}\text { Result to hospital } \\
\text { presentation (min) }\end{array}$ & Diagnosis \\
\hline 55 & 41 & 164 & 205 & 1.16 & NR & 398 & $\mathrm{AMI}$ \\
\hline 67 & 6 & 107 & 113 & $<0.10$ & 70 & 80 & UA \\
\hline 69 & 6 & 149 & 155 & 1.16 & 66 & 251 & AMI \\
\hline 70 & 163 & 86 & 249 & 3.66 & 49 & 357 & NSTEMI \\
\hline 85 & 12 & 101 & 113 & $<0.10$ & 43 & 1108 & UA \\
\hline 87 & 1188 & 66 & 1254 & 0.14 & 66 & 1312 & NSTEMI \\
\hline
\end{tabular}

eGFR = estimated glomerular filtration rate $\left(\mathrm{mL} / \mathrm{min} / 1.73 \mathrm{~m}^{2}\right)$; $\mathrm{NR}=$ not recorded; $\mathrm{AMI}=$ acute myocardial infarction; UA = unstable angina; $\mathrm{NSTEMI}=$ non-ST elevation myocardial infarction.

55-year-old patient), one cardiac arrest in a patient with known $\mathrm{CHD}$, and one episode of cardiogenic shock. During the follow-up period, 27 of 355 patients $(7.6 \%)$ had at least one ACS. The median time to presentation with the first ACS was 42 days (range, 0 days -498 days).

GP cohort: survey and linked data. For the 124 patients with both linked and survey data, there were 45 presentations to hospital, including 18 ACSs in 11 patients. Six occurred within 1 month of the cTn test, and in each case symptoms had commenced at least 48 hours before the test.

ED cohort. Three hundred and sixty-eight patients of the 1758 in the MIMIC dataset $(20.9 \%)$ received a discharge diagnosis of ACS, significantly more than the 13 patients $(3.7 \%)$ with an ACS in the GP cohort $(P<0.001)$. Most $(242 / 368,65.8 \%)$ were at high risk of ACS according to NHF/CSANZ criteria, with 114 $(31.0 \%)$ at intermediate risk and 12 $(3.3 \%)$ at low risk.

\section{Discussion}

This study found that most patients who underwent GP-initiated cTn testing had presented typical symptoms of coronary ischaemia and had clinical risk factors indicating intermediate or high risk of an ACS and associated adverse outcomes. ${ }^{15}$ While most results of GP-initiated cTn tests were negative, such a result did not rule out the possibility of an ACS, as $3.7 \%$ of patients
(13/355) were admitted to hospital with an ACS within 30 days of receiving a negative result. This approximates the 30-day event rate for patients presenting to an ED and classified as being of intermediate risk. ${ }^{15}$ In an ED, such patients would not be considered safe for discharge home until further investigations and monitoring had determined a lower risk level. ${ }^{15}$ The patients in our study, in contrast, would have been largely unmonitored in the community for some hours while awaiting their test results, as well as during the days following a negative result.

The finding that patients undergoing GP-initiated cTn testing were not low-risk was unexpected, and there may have been other factors not detected by the survey that reduced the risk status of patients. Obtaining comprehensive data on individual risk factors may have helped to resolve this question, including ECGs, quantitative blood pressure and lipid profiles, and the results of earlier invasive investigations for CHD. This information would also allow application of additional cardiovascular risk scoring tools and improve the generalisability of our study, although this would risk patient identification and reduced participation because of the longer survey duration.

Turnaround times in this study indicated that there was a substantial delay between presentation to a GP and cTn results becoming available. Particularly concerning was the median delay of more than 5 hours in those patients who were subsequently confirmed to have an ACS and who had presented within 48 hours of symptom onset, when the risk of complications is greatest. ${ }^{8}$ While the Royal Australian College of General Practitioners Standards ${ }^{20}$ require evidence of systems that ensure timely response to pathology results, there is evidence from the Threats to Australian Patient Safety (TAPS) study $^{21}$ and elsewhere 9 which suggests that this does not always occur.

GPs may not fully understand the limitations of cTn testing, as $23.4 \%$ of tests were ordered within 12 hours of symptom onset (Box 2), at which point the test may be insufficiently sensitive. While all major guidelines groups recommend serial testing to exclude ACS in this context, $^{4,8,22}$ no serial testing was performed by GPs in our study.

In many cases, the test result did not alter patient management. Some tests were clearly ordered in response to a patient request, and one GP commented that "the test was mainly arranged to satisfy the patient that this was unlikely cardiac". It is worth noting that a negative cTn test in this context may not have resolved patient anxiety, as many patients presented to a hospital within hours of receiving a negative test.

GPs are in a difficult situation. The consequences of missing an ACS diagnosis can be grave, yet there are no reliable clinical predictors of ACS, and primary care investigations have their limitations. At the 
same time, GPs have an important role as gatekeepers of the health system. ${ }^{23}$ Failure to accept any uncertainty may lead to unnecessary investigations and referrals, themselves potential causes of patient harm and unnecessary system costs. However, based on the results of our study, we concur with previous authors in this journal ${ }^{7,9}$ who have suggested that GPs should maintain a high threshold for requesting cTn testing and refer patients promptly to hospital for assessment when clinical features suggest a diagnosis of ACS. Possible ACS is one setting in which GPs can justifiably advise patients to present to a hospital, rather than undertaking investigations in primary care.

Competing interests: No relevant disclosures. -

๑ 2015 AMPCo Pty Ltd. Produced with Elsevier B.V. All rights reserved. 
1 Deloitte Access Economics. ACS in perspective: the importance of secondary prevention. Canberra: Deloitte Access Economics, 2011. https://www.deloitteaccesseconomics. com.au/uploads/File/Final\%20Report \%20ACS\%20in\%20Perspective\% 20Nov\%202011.pdf (accessed May 2015).

2 Briffa T, Hickling S, Knuiman M, et al. Long term survival after evidence based treatment of acute myocardial infarction and revascularisation: follow-up of population based Perth MONICA cohort, 1984-2005. BMJ 2009; 338: b36.

3 MacDonald PS, Newton PJ, Davidson PM. The SNAPSHOT ACS study: getting the big picture on acute coronary syndrome. Med J Aust 2013; 199: 147-148. https://www.mja.com.au/ journal/2013/199/3/snapshot-acsstudy-getting-big-picture-acutecoronary-syndrome

4 Anderson JL, Adams CD, Antman EA, et al. ACC/AHA 2007 guidelines for the management of patients with unstable angina/non-ST-elevation myocardial infarction: a report of the American College of Cardiology/American Heart Association task force on practice guidelines. J Am Coll Cardiol 2007; 50: el-el57.

5 Lozzi L, Carstensen S, Rasmussen H, Nelson G. Why do acute myocardial infarction patients not call an ambulance? An interview with patients presenting to hospital with acute myocardial infarction symptoms. Intern Med J 2005; 35: 668-671.

6 Bruyninckx R, Aertgeerts B, Bruyninckx $P$, Buntinx F. Signs and symptoms in diagnosing acute myocardial, infarction and acute coronary syndrome: a diagnostic meta-analysis. Br J Gen Prac 2008; 58: 105-11.

7 Parsonage WA, Cullen L, Younger JF. The approach to patients with possible cardiac chest pain. Med J Aust 2013; 199: 30-34. https://www.mja.com.au/ journal/2013/199/1/approach-patientspossible-cardiac-chest-pain

8 Chew DP, Aroney CN, Aylward PE, et al. 2011 addendum to the National Heart Foundation of Australia/Cardiac Society of Australia and New Zealand Guidelines for the Management of Acute Coronary Syndromes (ACS) 2006. Heart Lung Circ 2011; 20: 487-502.

9 Marshall GA, Wijeratne NG, Thomas D. Should general practitioners order troponin tests? Med J Aust 2014; 201: 155-157. https://www.mja.com.au/ journal/2014/201/3/should-generalpractitioners-order-troponin-tests

10 Taylor DM, Garewal D, Carter M, et al. Factors that impact upon the time to hospital presentation following the onset of chest pain. Emerg Med Australas 2005; 17: 204-211.

11 Hafiz AM, Naidu SS, DeLeon J, et al. Impact of first contact on symptom onset-to-door time in patients presenting for primary percutaneous coronary intervention. Am J Emerg Med 2013; 31: 922-927.

12 Law K, Elley CR, Tietjens J, Mann S. Troponin testing for chest pain in primary healthcare: a survey of its use by general practitioners in New Zealand. N Z Med J 2006; 119: U2082.

13 Tanner H, Larsen P, Lever N, Galletly D. Early recognition and early access for acute coronary syndromes in New Zealand: key links in the chain of survival. N Z Med J 2006; 119: U1927.

14 Aldous S, Gent P, McGeoch G, Nicholson D. The use of troponin in general practice. N Z Med J 2012; 125: 36-43.

15 Aroney CN, Aylward P, Kelly AM, et al. National Heart Foundation of Australia/Cardiac Society of Australia and New Zealand Guidelines for the management of acute coronary syndromes 2006. Med J Aust 2006; 184 (8 Suppl): SI-S30. https://www.mja. com.au/journal/2006/184/8/ guidelines-management-acute- coronary-syndromes-2006 (accessed Jul 2015).

16 Cullen L, Than M, Brown AFT, et al. Comprehensive standardized data definitions for acute coronary syndrome research in emergency departments in Australasia. Emerg Med Australas 2010; 22: 35-55.

17 National Centre for Classification in Health. The international statistical classification of diseases and related health problems, tenth revision, Australian modification (ICD-10-AM). 7th ed. Sydney: National Centre for Classification in Health, 2010.

18 National Centre for Classification in Health. The Australian classification of health interventions (ACHI). 7th ed. Sydney: National Centre for Classification in Health, 2010.

19 Macdonald SPJ, Nagree Y, Fatovich DM, et al. Serial multiple biomarkers in the assessment of suspected acute coronary syndrome: multiple infarct markers in chest pain (MIMIC) study. Emerg Med J 2013; 30: 149-154.

20 Royal Australian College of General Practitioners. Standards for general practices. 4th ed. Melbourne: Royal Australian College of General Practitioners, 2010. http://www.racgp. org.au/your-practice/standards/ standards4thedition/practiceservices/1-1/care-outside-normalopening-hours/ (accessed May 2015).

21 Makeham MA, Bridges-Webb C, Kidd MR. Lessons from the TAPS study. Managing investigation results - is your practice system safe? Aust Fam Physician 2008; 37: 625-626.

22 Thygesen K, Mair J, Giannitsis E, et al. How to use high-sensitivity cardiac troponins in acute cardiac care. Eur Heart J 2012; 33: 2252-2257.

23 Buntinx F, Mant D, Van den Bruel A, et al. Dealing with low-incidence serious diseases in general practice. $\mathrm{Br} J$ Gen Pract 2011; 61: 43-46. 